This item was submitted to Loughborough's Institutional Repository (https://dspace.lboro.ac.uk/) by the author and is made available under the following Creative Commons Licence conditions.

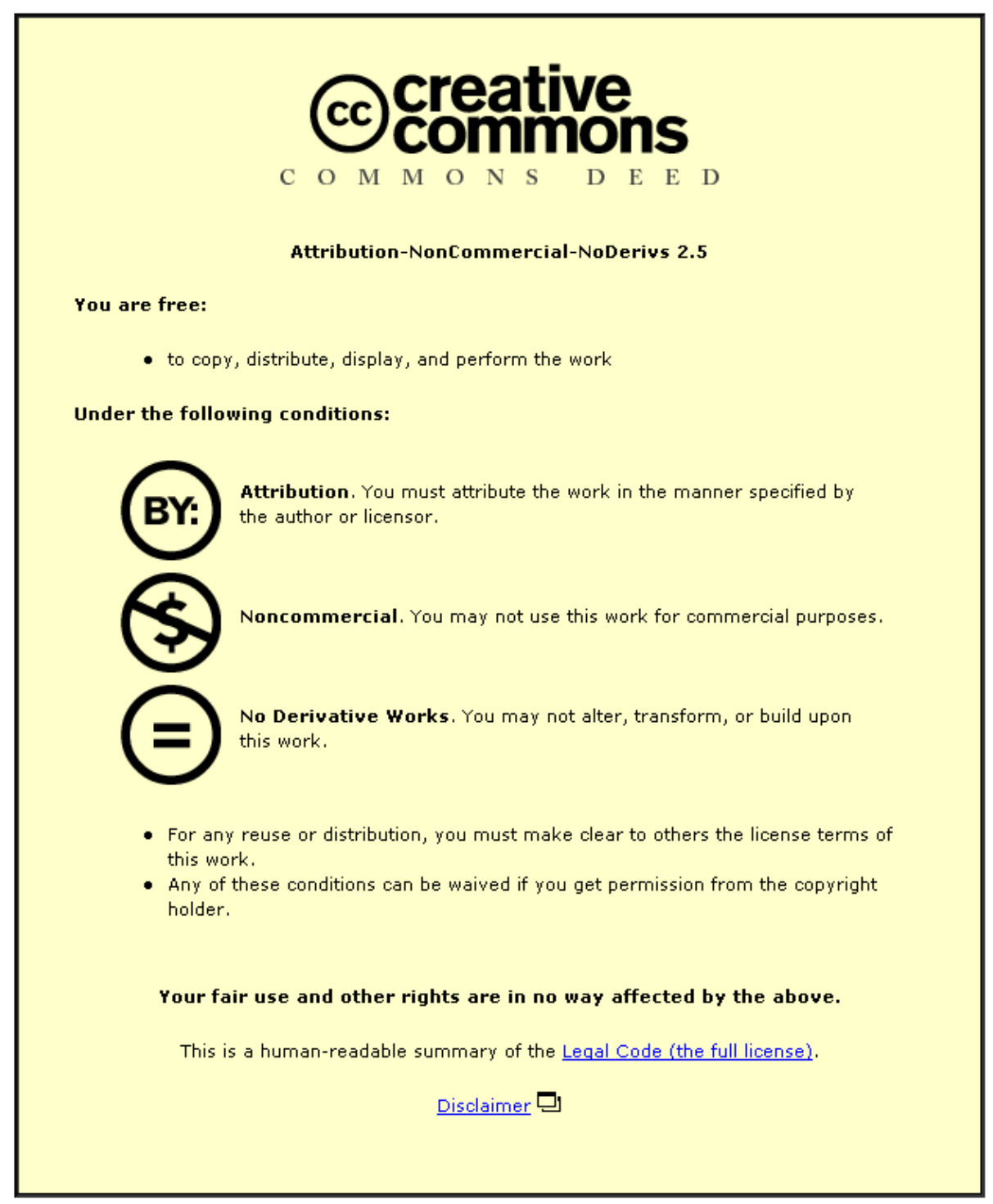

For the full text of this licence, please go to: http://creativecommons.org/licenses/by-nc-nd/2.5/ 


\title{
Parameter Estimation and Equalization Techniques for Communication Channels With Multipath and Multiple Frequency Offsets
}

\author{
S. Ahmed, S. Lambotharan, Member, IEEE, A. Jakobsson, Member, IEEE, and J. A. Chambers, Senior Member, IEEE
}

\begin{abstract}
We consider estimation of frequency offset (FO) and equalization of a wireless communication channel, within a general framework which allows for different frequency offsets for various multipaths. Such a scenario may arise due to different Doppler shifts associated with various multipaths, or in situations where multiple basestations are used to transmit identical information. For this general framework, we propose an approximative maximum-likelihood estimator exploiting the correlation property of the transmitted pilot signal. We further show that the conventional minimum mean-square error equalizer is computationally cumbersome, as the effective channel-convolution matrix changes deterministically between symbols, due to the multiple FOs. Exploiting the structural property of these variations, we propose a computationally efficient recursive algorithm for the equalizer design. Simulation results show that the proposed estimator is statistically efficient, as the mean-square estimation error attains the Cramér-Rao lower bound. Further, we show via extensive simulations that our proposed scheme significantly outperforms equalizers not employing FO estimation.
\end{abstract}

Index Terms-Frequency offset (FO) estimation, minimum mean-square error (MMSE) equalizer and complexity, wireless multipath channels.

\section{INTRODUCTION}

W IRELESS transmission is impaired by signal fading, interference, and additive measurement noises. If present, frequency offsets (FOs) can further seriously degrade the performance of a communication system. There are two main causes for FOs; poor synchronization between the transmitter and receiver carrier frequencies, or motion-induced Doppler shift, due to the relative motion between the mobile station and the local scatterer. Frequency estimation and tracking is crucial to enable accurate decoding of the transmitted information, and the FO estimation techniques for communication channels have been widely studied (see, e.g., [1] and [2]). However, the FOs have been assumed to be common for all multipaths. The FO and channel-estimation problem has also been addressed for a multiple-input and multiple-output (MIMO) system with distinct FOs for each transmit and receive antenna, but only for the case of a flat-fading channel [3]. In contrast to previous work, we address the FO problem for a single transmit and receive antenna,

Paper approved by V. A. Aalo, the Editor for Diversity and Fading Channel Theory of the IEEE Communications Society. Manuscript received July 1, 2003; revised February 25, 2004; June 16, 2004; and August 10, 2004.

S. Ahmed and J. Chambers are with the Centre of DSP, Cardiff School of Engineering, Cardiff University, Queen's Buildings, Cardiff CF24 OYF, U.K. (e-mail: ahmeds6@cardiff.ac.uk; chambersj@cardiff.ac.uk).

S. Lambotharan is with the Centre for DSP Research, Department of Electronic Engineering, Kings College London, London WC2R 2LS, U.K. (e-mail: s.lambotharan@kcl.ac.uk).

A. Jakobsson is with the Department of Electrical Engineering, Karlstad University, SE-651 88 Karlstad, Sweden (e-mail: andreas.jakobsson@kau.se).

Digital Object Identifier 10.1109/TCOMM.2004.841970 under a general framework allowing different FOs for each multipath. Such a scenario may arise when either the receiver or the transmitter moves at high speed, thereby resulting in distinct Doppler shifts for paths with different angles of arrival (AOAs). This effect can degrade the performance of the receiver significantly if not accounted for in the equalizer design. The organization of the paper is as follows. In the following section, the signal model is presented. Then, in Section III, we develop an approximate maximum-likelihood (AML) estimator for FOs and channel parameters, and compare its performance with the corresponding Cramér-Rao lower bound (CRLB). In Section IV, we study the minimum mean-square error (MMSE)-based equalizer and provide a computationally efficient algorithm for the equalizer design. Simulation results are given in Section V, followed by our conclusions in Section VI.

\section{Problem Statement}

Consider a communication system employing a single transmit and receive antenna, and assume that the signal has propagated through $L$ different paths, each having different FO and complex channel gain (CG). The received baseband signal can be written as

$$
r(n)=\bar{r}(n)+\eta(n)=\sum_{l=0}^{L-1} h_{l} x(n-l) e^{j 2 \pi f_{l} n}+\eta(n)
$$

where $h_{l}$ and $f_{l}$ denote, respectively, the unknown complex CG and the FO for the $l$ th channel tap. Herein, we assume that $h_{l}$ and $f_{l}$ are quasi-stationary, not changing significantly over the observed data frame, and only vary between data frames. Further, $x(n)$ is the transmitted signal with variance $\sigma_{x}^{2}$, and $\eta(n)$ is an additive circularly Gaussian distributed zero-mean white (complex) noise with variance $\sigma_{\eta}^{2}$. Let $\mathbf{X}_{l, n}$ denote the $N \times N$ diagonal matrix formed from the vector $\mathbf{x}_{l, n}$ along the diagonal, with

$$
\mathbf{x}_{l, n}=\left[\begin{array}{lll}
x(n-l) & \cdots & x(n-l-N+1)
\end{array}\right]^{T}
$$

where $N$ is the frame length, and $(\cdot)^{T}$ denotes the transpose. Further, let $\mathbf{e}_{l, n}$ model the FO for the $l$ th path

$$
\mathbf{e}_{l, n}=\left[\begin{array}{lll}
e^{j 2 \pi f_{l} n} & \cdots & e^{j 2 \pi f_{l}(n-N+1)}
\end{array}\right]^{T}
$$

and form the $N \times L$ matrix $\mathbf{V}_{n}$ as

$$
\mathbf{V}_{n} \triangleq\left[\begin{array}{lll}
\mathbf{v}_{0, n} & \cdots & \mathbf{v}_{L-1, n}
\end{array}\right]
$$

where $\mathbf{v}_{k, n}=\mathbf{x}_{k, n} \odot \mathbf{e}_{k, n}$, with $\odot$ denoting the SchurHadamard (elementwise) product. Hence, the frame constructed from $N$ consecutive received samples can be expressed as

$$
\begin{aligned}
\mathbf{r}_{n} & \triangleq\left[\begin{array}{lll}
r(n) & \cdots & r(n-N+1)
\end{array}\right]^{T} \\
& =\sum_{l=0}^{L-1} h_{l} \mathbf{X}_{l, n} \mathbf{e}_{l, n}+\boldsymbol{\eta}_{n} \\
& =\mathbf{V}_{n} \mathbf{h}_{L}+\boldsymbol{\eta}_{n}
\end{aligned}
$$


where $\mathbf{h}_{L}=\left[\begin{array}{lll}h_{0} & \cdots & h_{L-1}\end{array}\right]^{T}$ and $\boldsymbol{\eta}_{n}=\left[\begin{array}{llll}\eta(n) & \cdots & \eta(n-\end{array}\right.$ $N+1)]^{T}$. Here, the problem of interest is given $\mathbf{r}_{n}$ and $x(n)$, estimate the unknown parameter vector $\boldsymbol{\theta}=$ $\left[\begin{array}{llllll}h_{0} & \cdots & h_{L-1} & f_{0} & \cdots & f_{L-1}\end{array}\right]^{T} \triangleq\left[\mathbf{h}_{L}^{T} \mathbf{f}_{L}^{T}\right]^{T}$. We do not explicitly require the knowledge of the channel length, but knowledge on its upper bound is required. For an over-modeled system, the estimates of the additional channel coefficients would be close to zero, and in this way, the channel length can be determined. In the next section, we present a computationally efficient approach to estimate the unknown CGs and FOs.

\section{CG AND FO ESTIMATION}

In this section, we outline an AML estimator of the complex CGs and the FOs. Consider that the received signal, as expressed in (5), is only a function of the complex CGs and FOs. Thus, the log-likelihood function of the received signal frame can be expressed as (ignoring the constant terms)

$$
\ln p\left(\mathbf{r}_{n} \mid \boldsymbol{\theta}_{l}\right)=-\frac{1}{\sigma_{\eta}^{2}}\left(\mathbf{r}_{n}-\mathbf{V}_{n} \mathbf{h}_{L}\right)^{H}\left(\mathbf{r}_{n}-\mathbf{V}_{n} \mathbf{h}_{L}\right)
$$

where $(\cdot)^{H}$ denotes the conjugate transpose. Minimizing (6) with respect to $\mathbf{h}_{L}$ yields

$$
\hat{\mathbf{h}}_{L}=\left(\mathbf{V}_{n}^{H} \mathbf{V}_{n}\right)^{-1} \mathbf{V}_{n}^{H} \mathbf{r}_{n} \triangleq \mathbf{V}_{n}^{\dagger} \mathbf{r}_{n}
$$

where $\mathbf{V}_{n}^{\dagger}$ denotes the Moore-Penrose pseudoinverse. The FOs are estimated by minimizing the cost function, $J\left(\mathbf{f}_{L}\right)$, obtained by substituting (7) into (6)

$$
J\left(\mathbf{f}_{L}\right)=\mathbf{r}_{n}^{H} \mathbf{r}_{n}-\mathbf{r}_{n}^{H} \boldsymbol{\Pi}_{\mathbf{V}_{n}} \mathbf{r}_{n}
$$

where $\Pi_{\mathbf{V}_{n}}=\mathbf{V}_{n} \mathbf{V}_{n}^{\dagger}$ is the projection onto the range space of $\mathbf{V}_{n}$. By choosing the training sequence, $x(n)$, such that $E\left\{x^{*}(n-l) x(n-p)\right\}=\delta_{p-l}$, where $\delta_{k}$ denotes the Kronecker delta and $(\cdot)^{*}$ the conjugate, the $n$-dimensional minimization problem in (8) may be decoupled into $n$ one-dimensional problems, significantly reducing the complexity of the minimization. Note that $\mathbf{V}_{n}^{H} \mathbf{V}_{n}$ will be dominated by the large diagonal terms, with almost negligible contribution from the off-diagonal terms, if $x(n)$ is chosen as a pseudorandom sequence (as in the case of a training signal). Thus, $\mathbf{V}_{n}^{H} \mathbf{V}_{n} \approx \sum_{n=0}^{N-1}|x(n)|^{2} \mathbf{I} \triangleq \kappa \mathbf{I}$, where $\kappa$ is constant over the frame considered, enabling us to approximate the minimum of (8) as the maximum of

$$
\begin{aligned}
J^{\prime}\left(\mathbf{f}_{L}\right) & =\mathbf{r}^{H} \mathbf{V}_{n} \mathbf{V}_{n}^{H} \mathbf{r}_{n} \\
& =\sum_{p=0}^{L-1}\left|\sum_{n=0}^{N-1} r^{*}(n) x(n-p) e^{j 2 \pi f_{p} n}\right|^{2} .
\end{aligned}
$$

This is a difficult joint multidimensional optimization problem. We therefore consider the FO of an arbitrary individual path $s$ between a transmit and a receive antenna, for which the contribution to (9) is

$$
\begin{aligned}
\psi_{s}(n) & =r^{*}(n) x(n-s) e^{j 2 \pi f_{s} n} \\
& =Q_{s}+c_{1}(n)+c_{2}(n)
\end{aligned}
$$

where

$$
\begin{aligned}
Q_{s} & =h_{s}^{*}|x(n-s)|^{2} \\
c_{1}(n) & =\eta^{*}(n) x(n-s) e^{j 2 \pi f_{s} n} \\
c_{2}(n) & =\sum_{\substack{p=0 \\
p \neq s}}^{L-1} h_{p}^{*}(p) x^{*}(n-p) x(n-s) e^{j \Delta 2 \pi f_{p} n} \\
\Delta f_{p} & =f_{s}-f_{p} .
\end{aligned}
$$

Moreover, as $E\{x(n-u) x(n-v)\}=0$ for $u \neq v$, $\left|\sum_{n=0}^{N-1} \psi_{s}(n)\right|^{2} \approx N^{2}\left|h_{s}\right|^{2}|x(n-s)|^{4}+N \sum_{\substack{p=0 \\ p \neq s}}^{L-1}\left|h_{p}\right|^{2} \mid x(n-$ p) $\left.\right|^{2}|x(n-s)|^{2}=N^{2} \rho_{s}+N \rho_{i} \approx N^{2} \rho_{s}$ for large $N$. Therefore, provided that the ratio between the signal component for which the parameters are to be estimated, $\rho_{s}=\left|h_{s}\right|^{2}|x(n-s)|^{4}$, to the interfering components $\rho_{i}=\sum_{\substack{p=0 \\ p \neq s}}^{L-1}\left|h_{p}\right|^{2}|x(n-p)|^{2}|x(n-s)|^{2}$, is greater than $1 / N$, we could reduce the multidimensional joint maximization in (9) over all possible frequencies, to the maximization of the following for each individual frequency:

$$
\hat{f}_{s}=\arg \max _{f}\left|\sum_{n=0}^{N-1} r^{*}(n) x(n-s) e^{j 2 \pi f n}\right|^{2}
$$

which can be efficiently evaluated using the fast Fourier transform (FFT). Once the FOs are estimated, the CGs, $\mathbf{h}_{k}$, can be estimated using (7).

\section{MMSE EQUALIZER DESIGN}

\section{A. Equalizer for Channels Without FOs}

In this section, we initially consider the MMSE equalizer for the communication channel not affected by multiple FOs. Consider the received baseband signal in (1), for the case when $f_{p} \equiv 0$. For an equalizer of length $M$, the received signal vector can then be written as

$$
\tilde{\mathbf{r}}_{n}=\mathbf{H} \tilde{\mathbf{x}}_{n}+\tilde{\boldsymbol{\eta}}_{n}
$$

where $\tilde{\mathbf{r}}_{n}=\left[\begin{array}{lll}r(n) & \cdots & r(n-M+1)\end{array}\right]^{T}, \tilde{\mathbf{x}}_{n}=[x(n) \cdots x(n-$ $M-L+2)]^{T}, \tilde{\boldsymbol{\eta}}_{n}=[\eta(n) \cdots \eta(n-M+1)]^{T}$, and $\mathbf{H}$ is the $M \times(L+M-1)$ channel convolution matrix

$$
\mathbf{H}=\left[\begin{array}{cccc}
\mathbf{h}_{L}^{T} & 0 & \cdots & \mathbf{0} \\
0 & \mathbf{h}_{L}^{T} & 0 & \vdots \\
\vdots & 0 & \ddots & 0 \\
\mathbf{0} & \cdots & 0 & \mathbf{h}_{L}^{T}
\end{array}\right] .
$$

The MMSE equalizer is then found as [4], [5]

$$
\mathbf{w}_{o}=\left(\mathbf{H H}^{H}+\frac{\sigma_{n}^{2}}{\sigma_{x}^{2}} \mathbf{I}\right)^{-1} \mathbf{H z}_{v}
$$

where $\mathbf{z}_{v}$ is a coordinate vector, only containing a nonzero component at position $v$, i.e., $\mathbf{z}_{v}=\left[\begin{array}{lllllll}0 & \cdots & 0 & 1 & 0 & \cdots & 0\end{array}\right]^{T}$.

\section{B. Equalizer for Channels With FOs}

In the presence of FOs, the effective channel convolution matrix $\mathbf{H}$ will vary over time, and we modify (11) accordingly, yielding

$$
\tilde{\mathbf{r}}_{n}=\left(\mathbf{H} \odot \boldsymbol{\Gamma}_{L}\right) \tilde{\mathbf{x}}_{n}+\tilde{\boldsymbol{\eta}}_{n} \triangleq \mathbf{H}_{n} \tilde{\mathbf{x}}_{n}+\tilde{\boldsymbol{\eta}}_{n}
$$


where

$$
\begin{aligned}
\boldsymbol{\Gamma}_{L} & =\left[\begin{array}{cccc}
\boldsymbol{\gamma}_{n}^{T} & 0 & \cdots & \mathbf{0} \\
0 & \boldsymbol{\gamma}_{n-1}^{T} & 0 & \vdots \\
\vdots & 0 & \ddots & 0 \\
\mathbf{0} & \cdots & 0 & \boldsymbol{\gamma}_{n-M+1}^{T}
\end{array}\right] \\
\boldsymbol{\gamma}_{k} & =\left[\begin{array}{llll}
e^{j 2 \pi f_{0} k} & \cdots & e^{j 2 \pi f_{L-1} k}
\end{array}\right]^{T} .
\end{aligned}
$$

The optimum equalizer derived using (14), instead of (11), will thus yield

$$
\mathbf{w}_{o}=\left(\mathbf{H}_{n} \mathbf{H}_{n}^{H}+\frac{\sigma_{n}^{2}}{\sigma_{x}^{2}} \mathbf{I}\right)^{-1} \mathbf{H}_{n} \mathbf{z}_{v} \triangleq \mathbf{R}_{n}^{-1} \mathbf{H}_{n} \mathbf{z}_{v}
$$

which, due to the time-varying $\mathbf{H}_{n}$, will require the inversion of $\mathbf{R}_{n}$ for each symbol. Such an equalizer may be computationally infeasible. In the following, we propose a computationally efficient recursive scheme, not requiring the inversion of $\mathbf{R}_{n}$, by fully exploiting the movement of submatrices in $\mathbf{R}_{n}$. Generally for stationary channels, long equalizers provide better performance. However, for quasi-stationary channels, very long equalizers are not appropriate due to nonstationarity of the signal that enters into the equalizer. Here, for simplicity, we initially consider a simple example with only three channel taps and a length-three equalizer to illustrate the key ideas. Thus

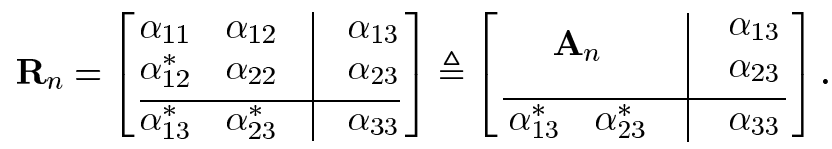

Note that, due to the structure of $\mathbf{R}_{n}$

$\mathbf{R}_{n+1}=\left[\begin{array}{l|ll}\beta_{11} & \beta_{12} & \beta_{13} \\ \hline \beta_{12}^{*} & \alpha_{11} & \alpha_{12} \\ \beta_{13}^{*} & \alpha_{12}^{*} & \alpha_{22}\end{array}\right]=\left[\begin{array}{l|ll}\beta_{11} & \beta_{12} & \beta_{13} \\ \hline \beta_{12}^{*} & \multicolumn{2}{|c}{\mathbf{A}_{n}} \\ \beta_{13}^{*} & & \end{array}\right]$

the Hermitian matrix $\mathbf{A}_{n}$ will appear in both $\mathbf{R}_{n}$ and $\mathbf{R}_{n+1}$. This structural property will hold for any number of channel coefficients and equalizer taps. For the general case, we write $\mathbf{R}_{n}$ as

$$
\mathbf{R}_{n}=\left[\begin{array}{cc}
\mathbf{A}_{n} & \mathbf{c}_{n} \\
\mathbf{c}_{n}^{H} & b_{n}
\end{array}\right]
$$

where $\mathbf{A}_{n} \in \mathbb{C}^{(M-1) \times(M-1)}, \mathbf{c}_{n} \in \mathbb{C}^{(M-1) \times 1}$, and $b_{n} \in \mathbb{R}$. Provided that the relevant inverses exist, the inverse of a general block matrix can be expressed as (19) or (20) (see, e.g., [6] and [7])

$$
\begin{aligned}
& {\left[\begin{array}{ll}
\mathbf{A} & \mathbf{C} \\
\mathbf{D} & \mathbf{B}
\end{array}\right]^{-1}=\left[\begin{array}{l}
\mathbf{I} \\
\mathbf{0}
\end{array}\right] \mathbf{A}^{-1}\left[\begin{array}{ll}
\mathbf{I} & \mathbf{0}
\end{array}\right]+\left[\begin{array}{c}
-\mathbf{A}^{-1} \mathbf{C} \\
\mathbf{I}
\end{array}\right]} \\
& \times\left(\mathbf{B}-\mathbf{D A}^{-1} \mathbf{C}\right)^{-1}\left[-\mathbf{D A}^{-1} \mathbf{I}\right] \\
& =\left[\begin{array}{l}
\mathbf{0} \\
\mathbf{I}
\end{array}\right] \mathbf{B}^{-1}\left[\begin{array}{ll}
\mathbf{0} & \mathbf{I}
\end{array}\right]+\left[\begin{array}{c}
\mathbf{I} \\
-\mathbf{B}^{-1} \mathbf{D}
\end{array}\right] \\
& \times\left(\mathbf{A}-\mathbf{C B}^{-1} \mathbf{D}\right)^{-1}\left[\mathbf{I} \quad-\mathbf{C B}^{-1}\right] \text {. }
\end{aligned}
$$

Thus, given $\mathbf{A}_{n}^{-1}$, one can easily obtain $\mathbf{R}_{n}^{-1}$ and $\mathbf{R}_{n+1}^{-1}$, avoiding the matrix inversion. As $\mathbf{A}_{n}$ does not appear in $\mathbf{R}_{n+2}$, it can not be used to find $\mathbf{R}_{n+2}^{-1}$, and the scheme so far only allows for a pairwise computational saving, still requiring the inversion of $\mathbf{A}_{n+1}$ to compute $\mathbf{R}_{n+2}^{-1}$ efficiently. However, further exploiting the structure of $\mathbf{R}_{n}$, one may compute $\mathbf{A}_{n+1}^{-1}$ efficiently from $\mathbf{R}_{n+1}^{-1}$ using the following lemma.

Lemma 1: Let

$$
\left[\begin{array}{ll}
\mathbf{H}_{11} & \mathbf{H}_{12} \\
\mathbf{H}_{21} & \mathbf{H}_{22}
\end{array}\right]=\left[\begin{array}{ll}
\mathbf{A}_{11} & \mathbf{A}_{12} \\
\mathbf{A}_{21} & \mathbf{A}_{22}
\end{array}\right]^{-1}
$$

where $\operatorname{dim}\left\{\mathbf{H}_{k l}\right\}=\operatorname{dim}\left\{\mathbf{A}_{k l}\right\}$. Then, provided that the relevant inverses exist, the inverse of matrix $\mathbf{A}_{11}$ can be written as the Schur complement of $\mathbf{H}_{22}$, i.e.,

$$
\mathbf{A}_{11}^{-1}=\mathbf{H}_{11}-\mathbf{H}_{12} \mathbf{H}_{22}^{-1} \mathbf{H}_{21} .
$$

The proof is given in the Appendix. At time $n$, let us find the inverse of submatrix $\mathbf{A}_{n}$, and use it, respectively, to find the inverses of $\mathbf{R}_{n}$ and $\mathbf{R}_{n+1}$ using (19) and (20). Since we know the inverse of $\mathbf{R}_{n+1}$, we find the inverse of the new top left-hand corner submatrix $\mathbf{A}_{n+1}$, using Lemma 1. Once found, the inverse of $\mathbf{A}_{n+1}$ can be used to compute the inverse of $\mathbf{R}_{n+2}$ using (20). We call this a forward and backward recursion method. Therefore, we need the explicit inverse of the matrix $\mathbf{A}_{n}$ only once, and thereafter, update the optimum equalizer for every symbol with the forward and backward recursions method using (20) and Lemma 1. We note that for our problem, $\mathbf{H}_{22}$ is a scalar.

\section{Simulations}

Initially, we assess the efficiency of the proposed AML estimator. A two-tap channel with FOs $f_{0}=0.003$ and $f_{1}=0.005$ is considered. This is reasonable, as the maximum Doppler shift for a vehicular speed of $250 \mathrm{~km} / \mathrm{h}$ (RA250 channels as defined in global systems for mobile communications (GSM) standards) at a carrier frequency of $900 \mathrm{MHz}$ is $1.3 \mathrm{KHz}$, which corresponds to 0.005 when normalized to the symbol rate of $270 \mathrm{KHz}$, as in GSM. The number of training samples is $N=100$. Figs. 1 and 2 show that the mean-square estimation error (MSEE) as obtained from $10^{4}$ Monte-Carlo simulations attains the CRLB, derived in the Appendix.

In order to demonstrate the benefits of employing FOs in equalization, we considered a two-path wireless communication channel and an equalizer of length 10 . We assume a quasi-stationary channel. The FOs have been initially set to 0.003 and 0.005 , and at every burst $n$, they have been changed according to the random-walk model, $f_{l}(n)=f_{l}(n-1)+0.0001 u(n)$, where $u(n)$ is a zero-mean real Gaussian noise with unity variance. The complex CGs $h_{0}$ and $h_{1}$ have been assumed to be constant throughout a burst, but change according to a Gaussian distribution between the frames. The training signal is assumed to have a binary alphabet, while the data symbols in the burst have been drawn from an 8-ary phase-shift keying (8-PSK) constellation. We considered three scenarios. In the first scenario, we set the FOs associated with the first and the second paths to $f_{0}=0.003$ and $f_{1}=0.005$, and designed a recursive equalizer, as explained previously. In the second scenario, we adopt a decision-directed scheme to equalize the channel effects, where the 


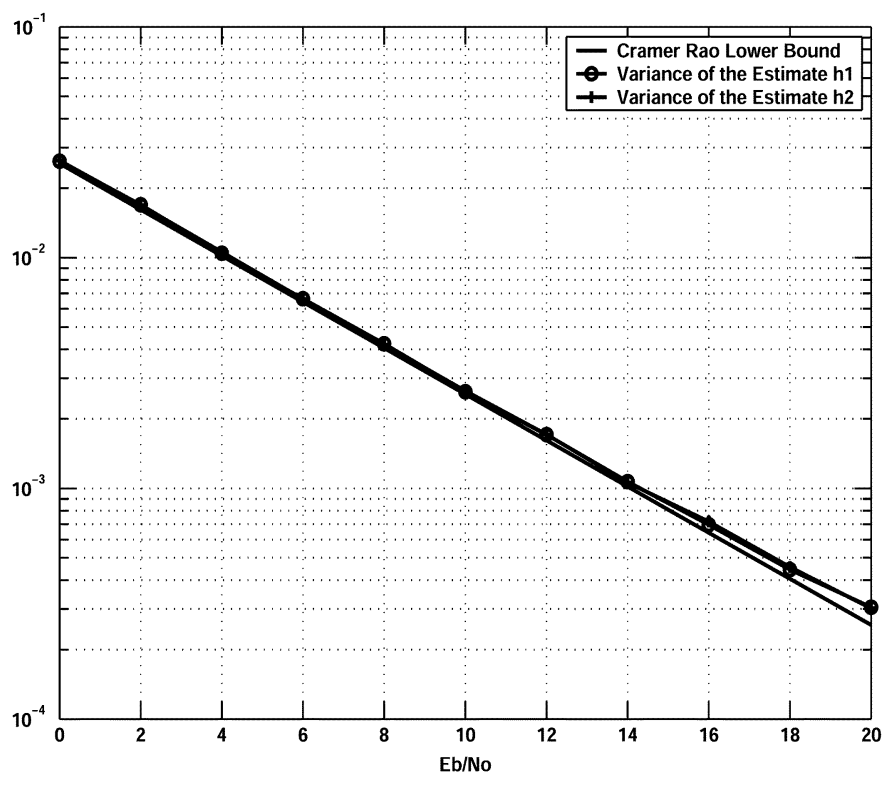

Fig. 1. Comparison of the MSEE of $h_{0}$ and $h_{1}$ with the CRLB.

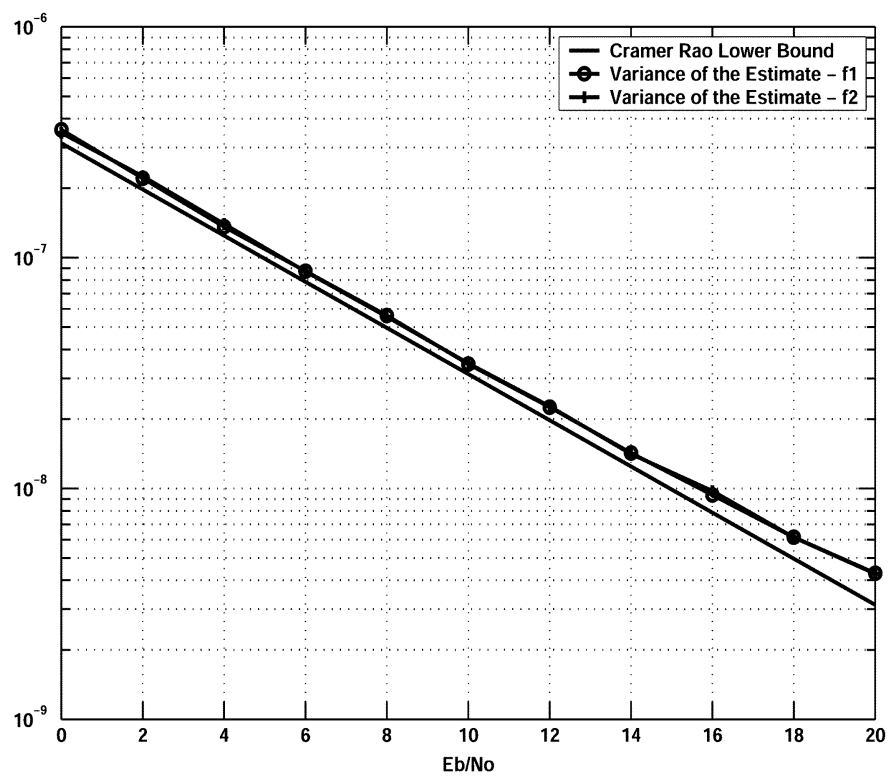

Fig. 2. Comparison of the MSEE of $f_{0}$ and $f_{1}$ with the CRLB.

least-mean square (LMS) algorithm is used to control the equalizer parameters. Here, the LMS equalizer is initialized with the correct MMSE solution, found at the end of the training interval. In the last scenario, an equalizer is designed ignoring the effect of FOs. The results depicted in Fig. 3 show the superior performance of our proposed scheme over the decision-directed scheme and an equalizer not considering the effect of FOs. In all the simulations, we considered a training signal of length 142 for the estimation of the channel parameters, including the FOs. The proposed scheme can also be employed in a GSM system. Here, the number of pilot symbols available in a burst is only 26 . With this training length, we can estimate the channel very efficiently, but to estimate FO, this length is insufficient. However, the performance of the FO estimator can be enhanced, by concatenating the pilot symbols from a number of recent past

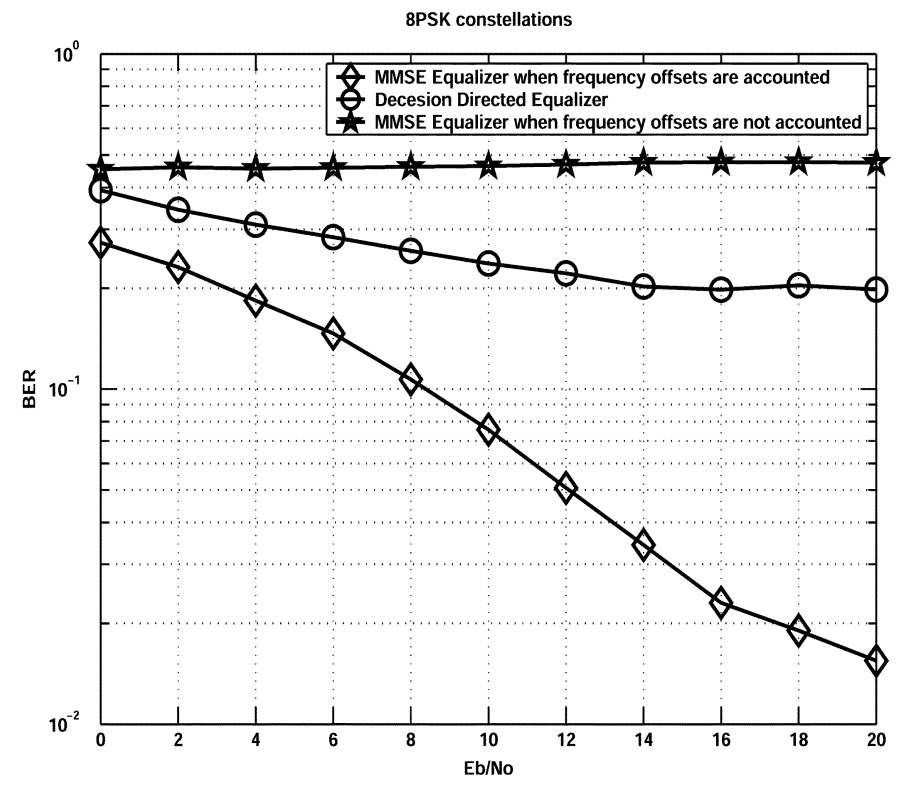

Fig. 3. BER performance for MMSE equalizers with and without FO estimation, and a decision-directed adaptive equalizer.

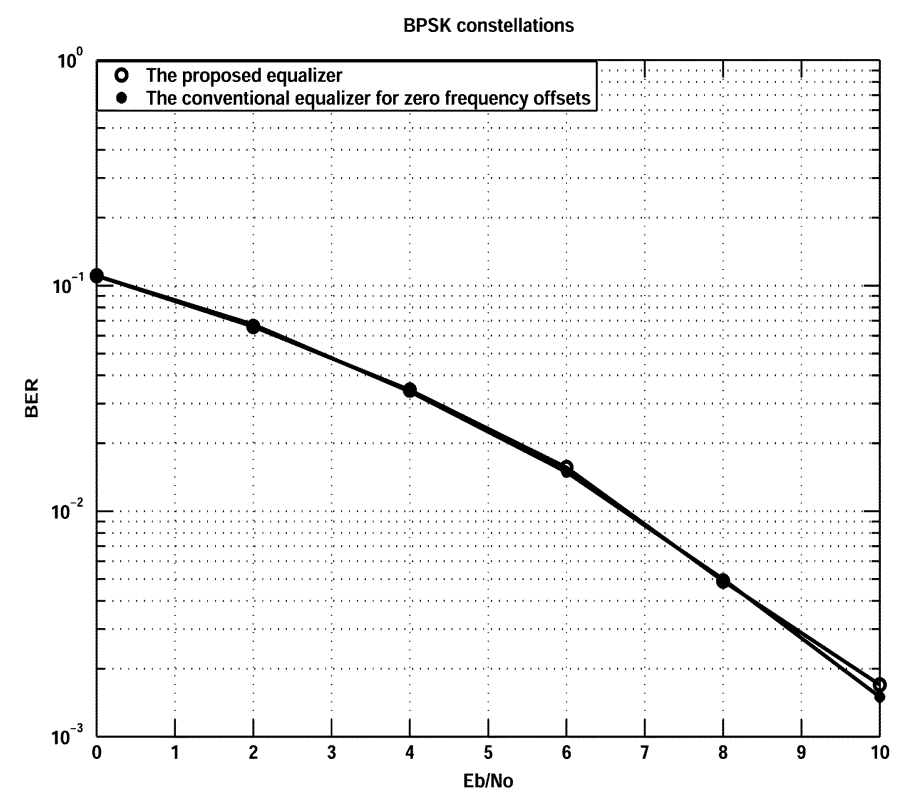

Fig. 4. BER performance of MMSE equalizers for a GSM system.

bursts. To illustrate this, we simulated GSM burst transmission through a two-path time-varying channel identical to that used in the previous simulation. A normal burst in GSM consists of 116 encrypted data symbols and 26 pilot symbols in the middle [8]. At every burst, the CGs $h_{0}$ and $h_{1}$ were estimated using the pilot symbols, but the FOs have been estimated using 142 symbols from the previous burst obtained with the retrieved data symbols together with the pilot symbols. In this way, to estimate frequency we used five previous successive bursts. To avoid occasional FO estimation error, we filtered the estimates through a moving median filter of length five. The bit-error rate (BER) performance of our proposed scheme is identical to the performance of a GSM communication channel without FOs (see Fig. 4). 


\section{CONCLUSION}

We considered estimation and equalization problems for a frequency-selective channel with distinct FOs for each path. This problem could arise when the receiver or transmitter moves with very high speed with different paths experiencing different Doppler shifts, due to different AOAs. By exploiting the correlation property of the transmitted pilot signal, we provided an AML estimator, and showed that the MSEE attains the CRLB. Since the effective channel convolution matrix varies between symbols due to multiple FOs, we provided a recursive scheme to design the optimum equalizer for every symbol. The simulation results demonstrate the superior performance of the proposed scheme over an equalization scheme that does not consider the effects of multiple FOs.

\section{APPENDIX I}

\section{DERIVATION OF CRLB}

In this appendix, we derive the CRLB for the problem at hand. Let $\overline{\mathbf{r}}(\boldsymbol{\theta})=\left[\begin{array}{lll}\bar{r}(n) & \cdots & \bar{r}(n-N+1)\end{array}\right]^{T}$ and $\boldsymbol{\theta}=\left[\Re\left(\mathbf{h}_{L}\right)^{T} \Im\left(\mathbf{h}_{L}\right)^{T} \mathbf{f}_{L}^{T}\right]^{T}$. Under the assumption that $\eta(t)$ is complex white Gaussian with zero mean and variance $\sigma_{\eta}^{2}$, the CRLB can be found using the Slepian-Bangs formula (see, e.g., [6])

$$
\left[\mathbf{P}_{\mathrm{CRLB}}^{-1}(\boldsymbol{\theta})\right]_{l, p}=2 \sigma_{\eta}^{-2} \Re\left\{\frac{\partial \overline{\mathbf{r}}^{H}(\boldsymbol{\theta})}{\partial \boldsymbol{\theta}_{\boldsymbol{l}}} \frac{\partial \overline{\mathbf{r}}(\boldsymbol{\theta})}{\partial \boldsymbol{\theta}_{p}}\right\}
$$

where $[\mathbf{A}]_{k, p}$ denotes the $(k, p)$ th element of $\mathbf{A}$, and $\mathbf{P} \triangleq E\left\{(\hat{\boldsymbol{\theta}}-\boldsymbol{\theta})(\hat{\boldsymbol{\theta}}-\boldsymbol{\theta})^{H}\right\} \geq \mathbf{P}_{\mathrm{CRLB}}$. We further note that $(\partial \bar{r}(n)) /\left(\partial h_{l}^{r}\right)=e^{j 2 \pi f_{l} n} \bar{r}(n-l),(\partial \bar{r}(n)) /\left(\partial h_{l}^{i}\right)=$ $j e^{j 2 \pi f_{l} n \bar{r}(n-l)}$, and $(\partial \bar{r}(n)) /\left(\partial f_{l}\right)=j 2 \pi n h_{l} e^{j 2 \pi f_{l} n} \bar{r}(n-l)$, where $h_{l}^{r}$ and $h_{l}^{i}$ are the $l$ th element of $\Re\left(\mathbf{h}_{L}\right)$ and $\Im\left(\mathbf{h}_{L}\right)$.

\section{APPENDIX II \\ PROOF OF LEMMA 1}

Using the block-matrix inversion lemma, as expressed in (19) and (20), (21) can be written as (see, e.g., [6] and [7])

$$
\begin{aligned}
& \mathbf{H}_{11}=\mathbf{A}_{11}^{-1}+\mathbf{A}_{11}^{-1} \mathbf{A}_{12} \boldsymbol{\Delta}_{\mathbf{A}}^{-1} \mathbf{A}_{21} \mathbf{A}_{11}^{-1} \\
& \mathbf{H}_{12}=-\mathbf{A}_{11}^{-1} \mathbf{A}_{12} \boldsymbol{\Delta}_{\mathbf{A}}^{-1} \\
& \mathbf{H}_{21}=-\boldsymbol{\Delta}_{\mathbf{A}}^{-1} \mathbf{A}_{21} \mathbf{A}_{11}^{-1} \\
& \mathbf{H}_{22}=\boldsymbol{\Delta}_{\mathbf{A}}^{-1}
\end{aligned}
$$

where $\boldsymbol{\Delta}_{\mathbf{A}}=\mathbf{A}_{22}-\mathbf{A}_{21} \mathbf{A}_{11}^{-1} \mathbf{A}_{12}$ is the Schur complement of $\mathbf{A}_{11}$. Substituting (27) into (26), and (25) into (24), yields

$$
\begin{aligned}
& \mathbf{H}_{21}=-\mathbf{H}_{22} \mathbf{A}_{21} \mathbf{A}_{11}^{-1} \\
& \mathbf{H}_{11}=\mathbf{A}_{11}^{-1}-\mathbf{H}_{12} \mathbf{A}_{21} \mathbf{A}_{11}^{-1} .
\end{aligned}
$$

Substituting (28) into (29) yields

$$
\mathbf{H}_{11}=\mathbf{A}_{11}^{-1}+\mathbf{H}_{12} \mathbf{H}_{22}^{-1} \mathbf{H}_{21}
$$

and hence (22), which concludes the proof.

\section{REFERENCES}

[1] H. Viswanathan and R. Krishnamoorthy, "A frequency offset estimation technique for frequency selective fading channels," IEEE Commun. Lett., vol. 5, pp. 166-168, Apr. 2001.

[2] K. E. Scott and E. B. Olasz, "Simultaneous clock phase and frequency offset estimation," IEEE Trans. Commun., vol. 43, pp. 2263-2270, Jul. 1995.

[3] O. Besson and P. Stoica, "On parameter estimation of flat fading channel with frequency offset," IEEE Trans. Signal Process., vol. 51, pp. 602-613, Mar. 2003.

[4] M. J. Heihhila, P. Komulainen, and J. Lilleberg, "Interference suppression in CDMA downlink through adaptive channel equalization," in Proc. IEEE Veh. Technol. Conf., vol. 2, Sep. 1999, pp. 978-982.

[5] S. M. Kay, Fundamentals of Statistical Signal Processing, Estimation Theory. Englewood Cliffs, NJ: Prentice-Hall, 1993.

[6] P. Stoica and R. Moses, Introduction to Spectral Analysis. Upper Saddle River, NJ: Prentice-Hall, 1997.

[7] T. Kailath and A. H. Sayed, Fast Reliable Algorithms for Matrices with Structure. Philadelphia, PA: SIAM, 1999.

[8] Multiplexing and Multiple Access on Radio Path, 3GPP TS 45.002, (V5.9.0), Apr. 2003. 